\title{
Pressão Positiva Contínua e Distúrbio Comportamental em Sono REM: A Propósito de um Caso Clínico
}

\section{Continuous Positive Airway Pressure and REM Sleep Behavior Disorder: A Case Report}

\author{
(D) Inês Carvalho ${ }^{1, *}$, Mafalda Ferreira ${ }^{2}$, (D) Joaquim Moita ${ }^{2}$, (D) Ana Catarina Brás ${ }^{3}$ \\ 1-Serviço de Neurologia / Centro Hospitalar e Universitário de Coimbra, Coimbra, Portugal. \\ 2-Centro de Medicina do Sono / Centro Hospitalar e Universitário de Coimbra, Coimbra, Portugal. \\ 3-Centro de Medicina do Sono e Serviço de Neurologia / Centro Hospitalar e Universitário de Coimbra, Coimbra, Portugal.
}

DOI: https://doi.org/10.46531/sinapse/IN/210008/2021

\section{Caso Clínico}

Mulher de 67 anos referenciada à Consulta de Patologia do Sono por roncopatia e fragmentação do sono. Desde os 45 anos apresentava sonhos vividos diários de conteúdo agressivo associados a movimentos bruscos noturnos.

Foi solicitada uma vídeo-polissonografia (vídeo-PSG) que revelou sono fragmentado com índice de microdespertares $51,3 /$ horas e RDI (respiratory disturbance index) de 19,2/horas. Foram observados comportamentos bruscos dos membros e discretos sonilóquios em sono REM, nomeadamente durante os eventos respiratórios (Vídeo 1), e uma perda parcial de atonia em sono REM (Fig. 1) com atividade motora fásica e tónica, achados compatíveis com distúrbio comportamental em sono REM (RSBD).

A doente foi diagnosticada com síndrome da apneia obstrutiva do sono (SAOS) moderada e RSBD isolado. Seis meses após iniciar terapêutica com autoCPAP (pressão positiva contínua nas vias respiratórias), mantinha sonhos de conteúdo agressivo, mas objetivou-se redução significativa da atividade motora noturna excessiva condicionando uma melhoria da qualidade do sono e RDI residual de 0,6/horas Foi iniciado

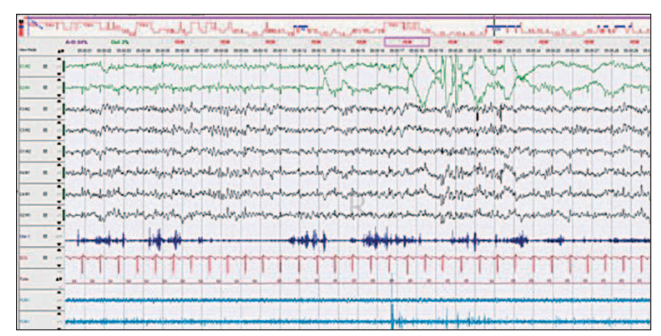

Figura 1. Traçado de Vídeo-PSG com 30 segundos de sono REM com perda de atonia.

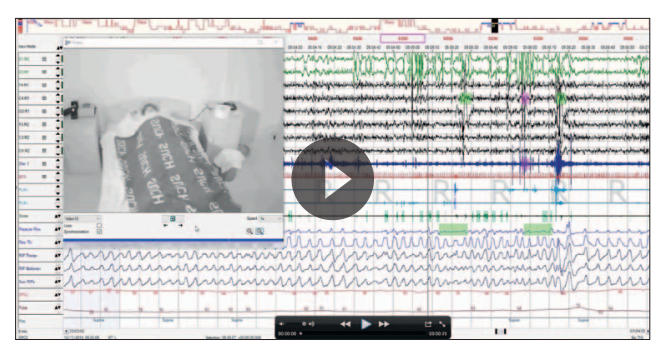

Vídeo 1. Traçado de Vídeo-PSG em Sono REM

Traçado de Vídeo-PSG em Sono REM confirmado pela presença de movimentos oculares rápidos (canais E1:M2 e E2:M1) e atividade de frequência mista no EEG (canais F4:M1 a O1:M2). Fisiologicamente deveria existir atonia muscular em Sono REM, contudo no mento (canal Chin1) observamos a presença de abundante atividade muscular tónica e fásica. Os comportamentos motores bruscos registados no vídeo ocorrem em relação temporal com o início de uma hipopneia (assinalada no canal Pressure Flow). A perda de atonia e os comportamentos motores observados em REM confirmam a presença de RSBD. clonazepam em doses baixas para controlo do conteúdo agressivo dos sonhos, com melhoria significativa. Cerca de 10 meses depois, a doente desenvolveu sintomatologia extrapiramidal (tremor em repouso e bradicinesia) com diagnóstico de doença de Parkinson (DP).

Informações/Informations: Imagem em Neurologia, publicado em Sinapse, Volume 21, Número 2, abril-junho 2021. Versão eletrónica em www.sinapse.pt

Image in Neurology, published in Sinapse, Volume 21, Number 2, April-June 2021. Electronic version in www.sinapse.pt (C) Autor (es) (ou seu (s) empregador (es)) e Sinapse 2021. Reutilização permitida de acordo com CC BY-NC.

Nenhuma reutilização comercial. (C) Author(s) (or their employer(s)) and Sinapse 2021. Re-use permitted under CC BYNC. No commercial re-use.

Palavras-chave:

Apneia Obstrutiva do Sono:

Perturbação do

Comportamento do Sono REM;

Pressão Positiva Contínua nas Vias Aéreas.

Keywords: Continuous Positive Airway Pressure;

REM Sleep Behavior Disorder; Sleep Apnea, Obstructive.

\author{
*Autor Correspondente / \\ Corresponding Author: \\ Inês Carvalho \\ Centro Hospitalar e \\ Universitário de Coimbra \\ Praceta Prof. Mota Pinto \\ 3004-561 Coimbra, Portugal \\ inesvideiradecarvalho@gmail.com
}

Recebido / Received: 2021-02-01 Aceite / Accepted: 2021-05-17 Publicado / Published: 2021-07-29 


\section{Discussão}

O RSBD é uma parassónia do sono REM que se caracteriza por perda de atonia permitindo uma encenação noturna real do conteúdo vivido e agressivo dos sonhos.

Atendendo à frequência de SAOS na população é importante reconhecê-la como possível comorbilidade em doentes com RSBD. Estas são as patologias do sono mais frequentemente encontradas na DP.

A curiosidade e particularidade deste caso clínico assenta na presença dos comportamentos motores e sonilóquios imediatamente após o início dos eventos respiratórios (hipopneias obstrutivas) em sono REM. A presença da SAOS poderia por si só justificar alguns dos movimentos bruscos, baseado na premissa da possibilidade de um "pseudo-RSBD" associado aos eventos respiratórios que condicionam microdespertares frequentes. No entanto, a perda de atonia em sono REM permitiu confirmar a presença de RBSD inicialmente considerado isolado, mas que posteriormente se revelou um sintoma pré-motor de DP.

SAOS e DP devem ser consideradas co-morbilidades: a referenciação à consulta foi motivada pelas queixas de roncopatia e sonhos vividos enquanto a sintomatologia extrapiramidal surgiu meses depois.

Neste caso, a terapêutica com autoCPAP foi suficiente para eliminar as manifestações motoras bruscas associadas aos eventos respiratórios, não só por eliminar as hipopneias obstrutivas, mas também por aliviar o seu efeito na fragmentação do sono. Os estudos sobre os efeitos do tratamento da SAOS na RSBD são escassos, mas existem relatos de melhoria subjetiva dos sintomas com introdução de CPAP.

A introdução de doses baixas de clonazepam nesta doente teve como principal objetivo atenuar a ativação cortical e límbica durante o sono REM, permitindo reduzir a vivacidade e conteúdo agressivo dos sonhos.

Neste caso clínico, o tratamento da SAOS foi fundamental para melhorar os sintomas motores noturnos, alertando para a importância da realização de uma vídeo-PSG no diagnóstico de distúrbios do movimento relacionados com o sono, eliminando os possíveis mimetizadores e confundidores e maximizando a abordagem terapêutica.

\section{Responsabilidades Éticas}

Conflitos de Interesse: Os autores declaram a inexistência de conflitos de interesse na realização do presente trabalho.

Fontes de Financiamento: Não existiram fontes externas de financiamento para a realização deste artigo.

Confidencialidade dos Dados: Os autores declaram ter seguido os protocolos da sua instituição acerca da publicação dos dados de doentes.

Consentimento: Consentimento do doente para publicação obtido.

Proveniência e Revisão por Pares: Não comissionado; revisão externa por pares.

\section{Ethical Disclosures}

Conflicts of Interest: The authors have no conflicts of interest to declare.

Financing Support: This work has not received any contribution, grant or scholarship.

Confidentiality of Data: The authors declare that they have followed the protocols of their work center on the publication of data from patients.

Patient Consent: Consent for publication was obtained.

Provenance and Peer Review: Not commissioned; externally peer reviewed.

\section{References / Referências}

1. Gabryelska A, Roguski A, Simpson G, Maschauer EL, Morrison I, Riha RL. Prevalence of obstructive sleep apnoea in REM behaviour disorder: response to continuous positive airway pressure therapy. Sleep Breath. 2018;22:825-30. doi: 10.1007/s11325-017-1563-9.

2. Gaig C, Iranzo A, Pujol M, Perez H, Santamaria J. Periodic limb movements during sleep mimicking REM sleep behavior disorder: A new form of periodic limb movement disorder. Sleep. 2017;40. doi: 10.1093/sleep/zsw063. 